\title{
New, insufficiently known, or rare for Lithuania species of beetles (Coleoptera) with some notes on ecology
}

\author{
Viktoras Pacevičius \\ Nature Research Centre, \\ Akademijos 2, \\ Vilnius 08412, Lithuania
}

\begin{abstract}
The paper presents faunistic data on 120 rare or insufficiently known beetle (Coleoptera) species, as well as one new species, Medon apicalis, for the fauna of Lithuania. In Europe, Agostenus costulatus, Anogcodes ferrugineus, Anogcodes rufiventris, Eubria palustris, Leiestes seminiger, Lissodema cursor, Mycetophagus ater, Orchesia undulata, Silvanoporus fagi are rare and local species; Anthobium unicolor, Drapetes mordelloides, Isoriphis melasoides, Oxyporus mannerheimi, Phyllotreta tetrastigma, Physoronia wajdelota, Plegaderus saucius, Nemozoma elongatum, Wanachia triguttata are rare and insufficiently known species. Saproxylic beetles such as Abdera flexuosa, Hallomenus axillaris, Hyleocoetus flabellicornis, Lissodema cursor, Orchesia undulata, Triphylus bicolor and Wanachia triguttata were found to be xylomicetophagous living in decaying wood overgrown with fungi or in fruiting bodies of tree fungi. New facts on ecology of three species were established: in 1994, 1996, and 1998, Calosoma auropunctatum population was recorded deep in mature forest; a case of high-density population of Hermaeophagus mercurialis was registered (approx. 600,000 specimens in an area of about $3.5 \mathrm{ha}$ ); and Boros schneideri was detected living in dead barkless pine trees in the middle of raised bogs. The locality where Medon apicalis was recorded in Lithuania is the easternmost edge of the species distribution range in Europe.
\end{abstract}

Keywords: insect fauna, saproxylic species, xylomicetophagous insects, Medon apicalis, Boros schneideri

\section{INTRODUCTION}

The paper provides data on insufficiently known, rare, and new beetle species in the Lithuanian fauna that the author collected from 1972 to 2018. Unlike the species within dragonfly (Odonata) or

\footnotetext{
* Corresponding author. Email: bareikis.col@gmail.com
}

butterfly and moth (Lepidoptera) orders, great majority of European species of which could be identified easily even during field survey, the identification of most beetle (Coleoptera) species is much more complicated and time-consuming, as well as tedious laboratory work. Despite this obstacle, it is important to know coleopteran species both for species diversity evaluations and for faunistic investigations of the country. 
The paper describes 120 easily identifiable (except for representatives of Altica, Carpophilus, Heterothops, Quedius, and Xylodromus genera) beetle species, including one new species for the Lithuanian fauna.

\section{MATERIALS AND METHODS}

Investigations were carried out in Moletai district and fragmentary observations were pursued all over the country. Field surveys were conducted using traditional beetle collection techniques. In addition, 18 standard funnel traps for Ips typographus control placed out in a mature coniferous forest compartment undergoing selective clearance were being inspected in 2016-2017. The tree-crown fauna was investigated by placing out plastic 3-5-section traps used for Ips typographus control, filled with beer- or wine-based baits. Such a method allowed collecting only a small number of species, with only one species (Cryptarcha strigata) published. From 2014 to 2018, saproxylic beetles were reared under laboratory conditions in hermetic boxes from deciduous wood of different state (hard, partly decomposed by rot fungi or microorganisms, completely decomposed, crushable between fingers); besides, xylomicetophagous beetles were reared from sponges and other fungi growing on trees as well as from parts of wood overgrown with fungi. Fully-fledged or crawling beetles were collected by vessels containing vinegar essence, by glue traps, or by making constant every $2-5$ day inspections of boxes.

The material collected is stored at the author's private collection. The species that are new for the Lithuanian fauna will be passed to the Kaunas T. Ivanauskas Zoological Museum.

The species were identified according to beetle (Coleoptera) identification guides (Burakowski, 1976; Lompe, 2018; Assing et al., 2011; Freude et al., 1965-1989; Warchałowski, 1971). Systematics and taxonomy are according to Tamutis et al. (2011) and adjusted as per Fauna Europaea Database. Colour photos were taken by a Canon ES 80d camera, using an Olympus SZX 10 binocular.

The material was collected and identified by the author, except for: R. F. - det. Romas Ferenca; V. M. - det. Vidmantas Monsevičius; V. T. - det. Vytautas Tamutis; V. P., V. T. - det. Viktoras Pacevičius, Vytautas Tamutis.

New species in the Lithuanian fauna are marked with an asterisk $\left(^{\star}\right)$, and the species assigned to Lithuania by different Palaearctic, European, and regional catalogues, without specifying the original source of information, are marked with a double asterisk ${ }^{* *}$. Many species were found in the environs of lakes, in which case the name of the lake is indicated as a locality where the species was recorded (e.g., Lake Baltis environs = Lake Baltis env.). The list of localities is given in the Table.

Table. List of localities

\begin{tabular}{ccc}
\hline Locality & Administrative district & Coordinates (latitude, longitude) \\
\hline Aidiečiai & Molètai & $55.29580,25.52069$ \\
\hline Ažuolijos Miškas f. & Utena & $55.44528,25.55963$ \\
\hline Lake Baltis env. & Molètai & $55.28862,25.58082$ \\
\hline Branišavos Miškas f. & Molètai & $55.35610,25.49839$ \\
\hline Degulių Miškas f. & Utena & $55.44932,25.54280$ \\
\hline Lake Dubulis env. & Molètai & $55.30966,25.57493$ \\
\hline Lake Ě̌erinis env. & Molètai & $55.30313,25.58973$ \\
\hline Gyvatraisčio Pelkè bog & Molètai & $55.35407,25.64747$ \\
\hline Kamšos Miškas f. & Kaunas & $54.90239,23.81587$ \\
\hline Kapiniškès & Varèna & $54.02662,24.29521$ \\
\hline
\end{tabular}


Table. (Continued)

\begin{tabular}{|c|c|c|}
\hline Locality & Administrative district & Coordinates (latitude, longitude) \\
\hline Kašeikiai env. & Molètai & $55.32831,25.59991$ \\
\hline Kavarskas & Anykščiai & $55.43085,24.93434$ \\
\hline Kazokų Miškas f. & Molètai & $55.12990,25.38446$ \\
\hline Kiauneliškis & Švenčionys & $55.29836,25.87095$ \\
\hline Kulautuvos Miškas f. & Kaunas & $54.94836,23.66652$ \\
\hline Kulionys env. & Molètai & $55.19221,25.68736$ \\
\hline Labanoras & Švenčionys & $55.27141,25.77297$ \\
\hline Lyduvėnai env. & Raseiniai & $55.49593,23.08258$ \\
\hline Lake Liūnelis env. & Molètai & $55.32079,25.58418$ \\
\hline Merkinė & Varèna & $54.16446,24.16777$ \\
\hline Milioniškès & Varèna & $54.19004,24.37780$ \\
\hline Lake Obelija env. & Lazdijai & $54.28660,23.82244$ \\
\hline Padaugupis & Kaunas & $55.01807,23.87264$ \\
\hline Rašia & Molètai & $55.19221,25.68796$ \\
\hline Seredžius & Jurbarkas & $55.08116,23.40689$ \\
\hline Suginčiai & Molètai & $55.33796,25.52439$ \\
\hline Šimonių Giria f. & Kupiškis & $55.72220,25.17536$ \\
\hline Tetervinio Pelkè bog & Zarasai & $55.69992,26.21369$ \\
\hline Uosa 1 & Prienai & $54.55800,23.91583$ \\
\hline Veliuona & Jurbarkas & $55.07741,23.28347$ \\
\hline Verkiai Park & Vilnius & $54.75069,25.30694$ \\
\hline Vilemai & Kaunas & $54.90797,23.67533$ \\
\hline Vilnius & Vilnius & $54.68242,25.26617$ \\
\hline Zervynos & Varèna & $54.10712,24.48756$ \\
\hline Lake Želva env. (1) & Molètai & $55.22387,25.57762$ \\
\hline Lake Želva env. (2) & Molètai & $55.32517,25.55592$ \\
\hline
\end{tabular}

\section{LIST OF SPECIES}

\section{CARABIDAE}

Cylindera germanica (Linnaeus, 1758)

Seredžius, 31.07.1990, 1 spec.; Uosa 1 , 24-25.07.2000, 3 spec.; Veliuona, 08.07.1987, 2 spec., attracted to light.

\section{Calosoma auropunctatum (Herbst, 1784)}

Suginčiai, 25.06-31.07.1972, about 5 spec., in the orchard; Lake Želva env. (2), 16.07.1994, 2 spec., 11.07.1996, 3 spec., 28.06.1998, 1 spec., attracted to light.

Note. In the environs of Lake Želva, the species was found in a sandy locality in the depth of a mixed forest, on a compartment line.
Carabus clathratus Linnaeus, 1761

Merkine, 16.06.1979, 1 spec.

Carabus convexus Fabricius, 1775

Rašia, 02-10.07.1977, about 7 spec.; Lake Želva env. (1), 30.04.2006, 1 spec., 18.04.2007, 2 spec.

\section{Carabus nitens Linnaeus, 1758}

Kavarskas, 1976, 1 spec.

\section{Carabus coriaceus Linnaeus, 1758}

Kamšos Miškas f., 22.07.2007, 1 spec.; Verkiai Park, 30.04.1991, 1 spec.

Note. The specimen found in Verkiai Park is the first record of the species in Vilnius environs.

Miscodera arctica (Paykull, 1758)

Kašeikès env., 2004, 1 spec. 
Laemostenus terricola (Herbst, 1784)

Suginčiai, 01-31.09.2007, 1 spec., 25.07.2011, 1 spec., in cellars.

Synuchus vivalis (Illiger, 1798)

Lake Želva env. (1), 01-10.08.2008, $1 \mathrm{spec}$.

Agonum ericeti (Panzer, 1809)

Suginčiai, 1976, 1 spec.; Tetervinio Pelkè bog 20-30.05.2002, 2 spec., in raised bogs.

Pterostichus aterrimus (Herbst, 1784)

Branišavos Miškas f., 05.05.2017, 2 spec.; Lake Ešerinis env., 15.08.2008, 1 spec.; Lake Liūnelis env. 01-10.05.2001, 1 spec., 19.05.2003, 3 spec., 24.04.2007, 1 spec., 08.08.2008, 1 spec. On the banks of water bodies, in moist areas.

Pterostchus macer (Marsham, 1802)

Kašeikiai, 2004, 1 spec.

Dolichus halensis (Schaller, 1783)

Kulautuvos Miškas f., 22.07.2003, 1 spec., attracted to light; Suginčiai, 11.07.2001, in an orchard.

Agostenus costulatus (Motschulsky, 1859)

Labanoras, 12-14.06.2003, 1 spec., in a gravel pit.

Cymindis angularis Gyllenhall, 1810

Suginčiai, September 2014, 1 spec.

\section{SPHAERITIDAE}

Sphaerites glabratus (Fabricius, 1792)

Lake Baltis env., 22.05.2017, 1 spec.; Lake Liūnelis env., 27.04.2007, 2 spec.; Suginčiai, 21.05.2007, 1 spec.; Lake Želva env. (2), 28.04.2007, 2 spec.

\section{HISTERIDAE}

\section{Plegaderus caesus (Herbst, 1792)}

Branišavos Miškas f., 25.05.2015, 1 spec.; 21.07.2015, 5 spec., - in the hollow of Fraxinus excelsior; Lake Želva env. (2), 29.07.2017, 1 spec., pheromone trap for Ips typographus control.

Plegaderus saucius Erichson, 1834

Suginčiai, 17.05.2015, 1 spec.

Plegaderus vulneratus (Panzer, 1797)

Suginčiai, May 2006, 1 spec.; Kulionys, 17.07.2017, 1 spec., pheromone trap for Ips typographus control.

Carcinops pumilio (Erichson, 1834)

Suginčiai, 03.07.2015, 13.08.2015, 01.06.2016, 03.07.2016, 25.07.2016, 04.08.2016, 06.08.2016,
$30.07 .2018,-11 \mathrm{spec}$. Indoors and in the nests of hollow-nesting birds.

Margarinotus bipustulatus (Schrank, 1781)

Lake Želva env. (1), 18.04.2007, 27.04.2007, -2 spec. On a sandy road.

Margarinotus obscurus (Kugellan, 1792)

Ažuolijos Miškas f., 23.05.2006, 1 spec.; Suginčiai, May 2006, 1 spec.

Hololepta plana (Schulzer, 1776)

Degulių Miškas f., 22.05.2014, 1 spec., on Picea abies trunk; Suginčiai, 16.03.2007, 1 spec., 20.04.2017, 2 spec., under the bark of Populus tremula.

\section{STAPHYLINIDAE}

\section{Acrulia inflata (Gyll, 1813)}

Lake Baltis env., 25.09.2017, 1 spec., in $H y$ pholoma spp. mycelium. One report earlier (Monsevičius, 2013).

Xylodromus brunnipennis (Stephens, 1832)

Suginčiai 16.05.2017, 1 spec., in a nest (det. V. M.).

${ }^{*}$ Xylodromus depressus (Gravenhorst, 1802) (Fig. 1)

Suginčiai, 31.08.2015, 2 spec., 19.09.2016, 1 spec. (det. V. M.).

Anthobium unicolor (Marasham, 1802)

Suginčiai, 02.04.2015, 1 spec., 28.05.2016, 1 spec., 06.07.2018, $1 \mathrm{spec}$.

Anthophagus angusticollis (Mannerheim, 1830)

Lyduvėnai, 26.05.2018, 6 spec.; Verkiai Park, 28.05.2015, 1 spec.

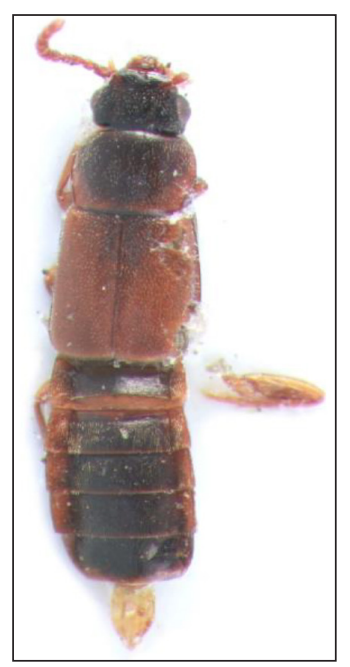

Fig. 1. Xylodromus depressus 
Tachinus marginatus (Fabricius, 1793)

Branišavos Miškas f., 21.09.2017, 1 spec.; Suginčiai, 25.04.2015, 1 spec., 28.04.2015, 1 spec., 15.04.2016, 1 spec., 01.05.2016, 1 spec. Near tree sap and under deciduous tree bark.

Trichophyia pilicornis (Gyllenhall, 1810)

Suginčiai, 01.05.2007, 1 spec.; Lake Želva env. (1), 29.05.2017, 1 spec.

Lomechusa emarginata (Paykull, 1789)

Kulionys env., 16.04.2007, 1 spec., on Formica spp. anthill.

Anomognathus cuspidatus (Erichson, 1839)

Branišavos Miškas f., 30.05.2016, about 10 spec., under the bark of Populus tremula.

Oxyporus mannerheimi (Gyllenhal, 1827)

Lake Dubulis env., 27.07.2017, 1 spec., 25.09.2017, 1 spec., 24.08.2018, 1 spec.; Lake Ešerinis env., 05.09.2004, 1 spec.; Lake Liūnelis env., 09.06.2003, 1 spec.; Kašeikiai env., 03.06.2003, 2 spec.; Lake Želva env. (2), 08.08.2013, 1 spec. In fungi Boletus spp., Leccinium spp.

Dianous coerulescens (Gyllenhal, 1810)

Suginčiai, 2006, 1 spec.

Rugilus angustatus (Geoffroy, 1785)

Branišavos Miškas f., 28.05.2016, 1 spec.; Suginčiai 09.06.2017, 1 spec.

${ }^{\star}$ Medon apicalis (Kraatz, 1857) (Fig. 2)

Suginčiai, 29.05.2016, 1 spec., 01.06.2016, 2 spec., 29.05.2017, 1 spec. (det. V. M.). Flying in the daytime, of which one near Lasius spp. anthill.

Diagnosis: Resembles Medon ripicola (Kr., 1854) and M. brunneus (Er., 1839). Size: 3.5$4.7 \mathrm{~mm}$. The main external morphological traits: foreback smaller than head, narrower than elytra at shoulder level; upper part brown, only head and occasionally elytron tips blackish; posterior edge of 5th sternite curved in the middle, covered with long black hairs. (Lompe A., after Lohse G. E.)

Medon castaneus (Gravenhorst, 1802)

Suginčiai, 13.05.2017, 1 spec.

Ocypus ophthalmicus (Scopoli, 1763)

Kazokų Miškas f., 05.07.2017, 1 spec.

${ }^{\star *}$ Heterothops dissimilis (Gravenhorst, 1802) (Fig. 3)

Suginčiai, June 2004, 1 spec., 01.06.2016, 1 spec. (det. V. M.).
Velleius dilatatus (Fabricius, 1787)

Suginčiai, July 1986, 1 spec., 19.07.2007, 1 spec., 09.07.2016, 1 spec.

Quedius brevicornis (Thomson, 1860)

Suginčiai, 20.04.2016, 1 spec., 15.06.2017,

1 spec. In the nests of hollow-nesting birds.

Quedius lucidulus Erichson, 1869

Branišavos Miškas f., 16.04.2016, 1 spec., 07.04.2017, 1 spec. (det. V. M.).

Quedius scitus (Gravenhorst, 1806)

Suginčiai, 23.09.2014, 1 spec., 17.08.2015, 1 spec., 01.05-10.07.2016, 1 spec.

\section{Quedius vexans Eppelsheim, 1881}

Suginčiai, 15.07.2007, 1 spec., 26.07.2014, 1 spec., 01.05.2016, 1 spec. In bird nests.

Acylophorus wagenschieberi Kiesenwetter, 1850

Ažuolijos Miškas f., 21.05.2007, 1 spec., attracted to light.

\section{PSEPHENIDAE}

\section{Eubria palustris Germar, 1818}

Lake Baltis env., 21.07.2017, 1 spec., 27.07.2017, $10 \mathrm{spec}$. On a floodplain of a forest stream. One report (Ferenca, 1988).

\section{EUCNEMIDAE}

\section{Isoriphis melasoides Castelnau, 1825}

Branišavos Miškas f., 03-19.07.2015, 2 spec., reared from trunks of deciduous trees. One report (Ferenca \& Tamutis, 2009).

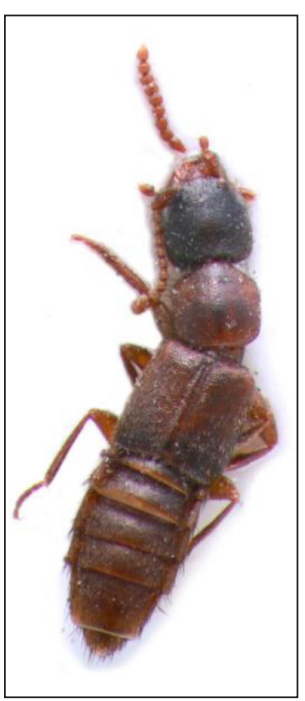

Fig. 2. Medon apicalis

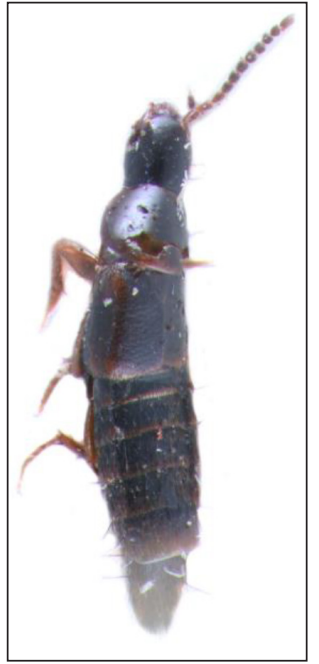

Fig. 3. Heterothops dissimilis 
Melasis buprestoides (Linnaeus, 1761)

Branišavos Miškas f., 01.06-12.07.2015, about 10 spec., reared from Alnus spp., Betula spp., Quercus robur; Lake Baltis env., 0517.05.2018, 4 spec., reared from Betula spp.

Otho spondyloides (Germar, 1818)

Lake Baltis env., 23-30.07.2018, 1 spec., reared from Betula spp. One report (Pacevičius, 2017).

\section{DASCILLIDAE}

Dascillus cervinus (Linnaeus, 1758)

Suginčiai, June 2004, 3 spec.; Lake Želva env. (2), 10.07.2017, 2 spec.

\section{ELATERIDAE}

Drapetes mordelloides (Holst, 1789)

Suginčiai, 17.07.2007, 1 spec., 20.06.2008, 1 spec., 21-22.06.2014, 2 spec. In decaying oak logs.

\section{LYMEXYLIDAE}

Hyleocoetus flabellicornis (Schneider, 1791)

Lake Želva env., 24.04.2014, 1 spec., flying near a decaying fir tree log.

\section{TROGOSSITIDAE}

Nemozoma elongatum (Linnaeus, 1761)

Branišavos Miškas f., 28.04-03.06.2015, 7 spec., reared from Alnus spp., Betula spp., 04.06.2017, 1 spec., on Populus tremula log.

\section{SILVANIDAE}

Ahasverus advena (Waltl, 1864)

Suginčiai, 27.01.2017, 1 dead spec., in a barn.

Oryzaephilus surinamensis (Linnaeus, 1758)

Suginčiai, 03.07.2000, 1 spec.

Dendrophagus crenatus (Paykull, 1799)

Kulionys, 08.08.2008, 1 spec., 31.08.2015, 1 spec., 01.04.2016, 1 spec.; Lake Liūnelis 26.11.2012, 4 spec.; Suginčiai 25.05.2006, 1 spec., September 2007, 1 spec., 10.06.2017, 1 spec. Under the bark of Pinus sylvestris, Picea abies, Populus tremula, Salix caprea.

Silvanus bidentatus (Fabricius, 1792)

Suginčiai, 19.04.2015, 4 spec., 20.05.2017, 2 spec., 20.06.2017, 1 spec., under the bark of Populus tremula, 23.05.2017, 2 spec., reared from Populus tremula.
Silvanus unidentatus (Olivier, 1790)

Branišavos Miškas f., 29.07.2016, 1 spec., 29.05.2017, 1 spec., reared from deciduous trees; Suginčiai, 13.07.2015, 1 spec., on Quercus robur, 20.05-12.06.2017, 6 spec., reared from Quercus robur, Populus tremula, Picea abies(?).

Silvanoporus fagi (Guerin-Meneville, 1844)

Suginčiai, 20.05.2017, 2 spec., in a stack of logs.

\section{LAEMOPHLOEIDAE}

Cryptolestes pusillus (Schonherr, 1817)

Branišavos Miškas f., 28-31.07.2018, 2 spec., reared from Quercus robur. One report (Monsevičius, 2013).

\section{NITIDULIDAE}

Carpophilus marginellus (Motschulsky, 1898)

Suginčiai, 17.07.2007, 1 spec., 27.07.2014, 1 spec. Near oak sap.

Amphotis marginata (Fabricius, 1781)

Suginčiai, 28.06.2014, 1 spec.; Suginčiai, 21.06.2016, 1 spec.

\section{Ipidia binotata (Reitter, 1875)}

Suginčiai, 06.09.2014, 1 spec.; 08.04.2018, 1 spec., 16.04.2018, 4 spec. Under the bark of Picea abies.

Physoronia wajdelota (Wankovicz, 1869)

Suginčiai, 18.08.2014, 1 spec., in a gilled mushroom on Alnus spp.

Thalycra fervida (Olivier, 1790)

Lake Dubulis env., 10.08.2017, 1 spec., in a pheromone trap for Ips typographus control; Kašeikiai env., 14.07.2014, 3 spec., caught by a sweep net in a mesophytic meadow with shrubs; Kulionys env., 2012, 1 spec.; Suginčiai, 16.06.2016, 1 spec., 22.06.2014, 1 spec., 03.07.2014, 3 spec., 23.07.2014, 3 spec., in mesophytic meadows and at dusk near shrubs; Lake Želva env. (2), 28.05.2016, 1 spec.

Cryptarcha strigata (Fabricius, 1787)

Branišavos Miškas f., 13.07.2015, 2 spec., 16.07.2015, 2 spec. In tree crown traps.

Glischrochilus quadrisignatus (Say, 1835)

Branišavos Miškas f., 29.06.2015, 13.07.2015, 06.08.2015, 14-25.08.2018, 8 spec., reared from deciduous stumps overgrown with fungi; 
Suginčiai, 09.06.2006, 15.07.2007, 20.07.2011, 01-10.07.2014,07.06.2015,25-30.06.2015, about 17 spec., mostly under the bark of Populus tremula, Quercus robur and near oak sap; Vilnius, July 2011, 1 spec., on a sponge on Salix spp.

\section{ENDOMYCHIDAE}

\section{Leiestes seminiger (Gyllenhall, 1808)}

Branišavos Miškas f., 07.07.2015, 23.08.2015, 05.05.2016, 3 spec., reared from deciduous trees; Suginčiai, 20.05.2017, 25.05.2017, 04.05.2018, 4 spec., on fir and asp logs; Lake Želva env. (2), $10.08 .2017,1 \mathrm{spec}$., in a pheromone trap for Ips typographus control.

\section{Mycetina cruciata (Schaller, 1783)}

Suginčiai, 01-15.09.2018, 5 spec., in a heap of decaying oak logs.

\section{COCCINELLIDAE}

Scymnus ferrugatus (Moll, 1785)

Kulionys env, 16.07.2015, 1 spec. (det. V. M.).

Hyperaspis pseudopustulata Mulsant, 1853

Aidiečiai, 12.05.2016, 1 spec., in a mesophytic meadow.

\section{Sospita vigintiguttata (Linnaeus, 1758)}

Branišavos Miškas f., 21.07.2015, 2 spec., 22.06.2016, 1 spec.; Kulionys env., 26.06.2016, 27.09.2016, 2 spec.; Suginčiai, 02.04.2017, 1 spec.; Lake Želva env. (1), 06.07.2014, 1 spec., 18.07.2014, $1 \mathrm{spec}$. In crowns of shrubs and trees.

Vibidia duodecimguttata (Poda, 1761)

Branišavos Miškas f., 16.05.2017, 1 spec.; Suginčiai, 16.05.2016, 1 spec. In tree crowns.

\section{MYCETOPHAGIDAE}

Triphyllus bicolor (Fabricius, 1777)

Branišavos Miškas f., 11.10.2014, 2 spec., in a pitfall (Barber) trap. One report (Ferenca et al., 2002).

\section{Mycetophagus ater (Reitter, 1879)}

Branišavos Miškas f., 11.05.2016, 1 spec., reared from a Fomes fomentarius berry bush; Lake Želva env. (2), 1 spec., in a pheromone trap for Ips typographus control.

Mycetophagus populi (Fabricius, 1798)

Lake Dubulis env., 02.07.2017, 1 spec., 0311.08.2017, 2 spec., in a pheromone trap for longhorn beetle (Cerambycidae) control; Suginčiai,
05.05.2017, 1 spec., 09.07.2017, 1 spec., reared from a Gonoderma lucidum berry bush.

\section{TETRATOMIDAE}

Hallomenus axillaris (Illiger, 1807)

Suginčiai, 25.07.2018, 1 spec., 18-20.08.2018, 2 spec., reared from Betula spp., Quercus robour, Lake Želva env. $(1,2), 15.06 .2015,21.07 .2015$, 27.07.2015, 06.08.2015, 5 spec., reared from Alnus spp., Betula spp., 03.06.2017, 1 spec., in a pheromone trap for Ips typographus control.

\section{MELANDRYIDAE}

\section{Orchesia minor Walker, 1836}

Branišavos Miškas f., 13.06.2018, 1 spec., reared from Alnus spp.

\section{Orchesia undulata Kraatz, 1853}

Branišavos Miškas f., 13.10.2014, 1 spec., in a hollow in Populus tremula; Lake Želva env. (2), 21.07.2017, 1 spec., in a pheromone trap for Ips typographus control, 01-19.09.2018, 1 spec., reared from Alnuss spp.

${ }^{*}$ Abdera flexuosa (Paykull, 1799) (Fig. 4)

Lake Želva env. (1, 2), May-June 2007, 2 spec., 25.05.2015, 1 spec., 03.06.2015, 1 spec., 21.06.2018, 1 spec., under the bark of Betula spp. and reared from decaying Alnus spp., Betula spp. (det. V. P., V. T.).

Morphological traits: foreback blackish, wide upper and lower edges light; antennae blackish in the middle, posterior and 2-3 base segments light; elytra with two black broken transverse bands (Duff, 2012).

Wanachia triguttata (Gyllenhall, 1810)

Kulionys env., 16.06.2014, 1 spec., in a sweep net (det. R. F.). One record (Ferenca et al., 2002).

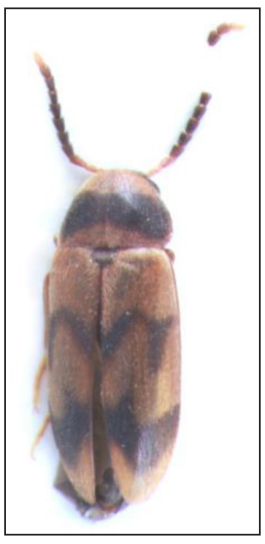

Fig. 4. Abdera flexuosa 
Zilora obscura (Fabricius, 1794)

Šimonių Giria f., 28.05.2013, 1 spec.

\section{RHIPIPHORIDAE}

Metoecus paradoxus (Linnaeus, 1761)

Kulautuvos Miškas f., 23.07.2003, 1 spec., attracted to light; Suginčiai, 24-27.07.2018, 1 spec., 06-09.08.2018, 1 spec., in a hollow in Salix fragilis.

\section{ZOPHERIDAE}

Colydium filiforme Fabricius, 1792

Ažuolijos Miškas f., 01-31.05.2007, 1 spec., on a decaying Quercus robur stump; Suginčiai, 23.05.2006, 1 spec., under the bark of Qercus robur.

\section{TENEBRIONIDAE}

Blaps mortisaga (Linnaeus, 1758)

Suginčiai, 1982, 1 spec.; Milioniškès, 22.09.2001, 1 spec. In cellars.

Mycetochara axillaris (Paykull, 1799)

Suginčiai, 20.06.2016, 1 spec.

Mycetochara humeralis (Fabricius, 1787)

Suginčiai, 21.05-30.06.2007, 1 spec.

Hypophloeus bicolor (Olivier, 1790)

Branišavos Miškas f., 24.05.2015, 2 spec., reared from Betula spp.; Suginčiai, 05.06.2006, 5 spec., under the bark of deciduous trees.

Scaphidema metallicum (Fabricius, 1793)

Suginčiai, 12.06.2016, 1 spec., in Picea abies crown (det. V. T.).

\section{OEDEMERIDAE}

Anogcodes ferrugineus (Schrank, 1776)

Lake Dubulis env., 14-23.06.2017, 3 spec., in a moist meadow on a lakeshore.

Anogcodes melanurus (Fabricius, 1787)

Vilnius, June 2000, 1 spec.

Anogcodes rufiventris (Scopoli, 1763)

Zervynos, 18.06.1985, 1 spec.

Oedemera femorata (Scopoli, 1763)

Padaugupis, 21.07.2007, 1 spec., attracted to light.

\section{BORIDAE}

Boros schneideri (Panzer, 1795)

Gyvatraisčio Pelkè bog, 21-31.06.2017, 2 spec.; Kiauneliškis, 26.10.2013, 3 spec.; Suginčiai, 11.08.2017, 1 spec.
Note. The findings from Gyvatraisčio Pelkè bog and Suginčiai were reared in laboratory conditions from dead, barkless pine trees from an open area of the bog.

SALPINGIDAE

Lissodema cursor (Gyllenhall, 1813)

Branišavos Miškas f., 09.07.2016, 12.07.2016, 2 spec., reared from a dead Quercus robour. One report (Tamutis et al., 2008).

Sphaeriestes bimaculatus (Gyllenhall, 1810) Lake Baltis env., 14.05.2017, 1 spec., flying in a clearing of a fir sapling forest, near a marsh.

Colposis mutilatus (Beck, 1817)

Kulionys, 16.04.2007, 1 spec., under the bark of Quercus robur; Suginčiai, 02.05.2017, 2 spec., on the wings; Lake Želva env. (2), 21.07.2017, 27.07.2017. 2 spec., in a pheromone trap for Ips typographus control.

Rabdocerus foveolatus (Ljungh, 1823)

Branišavos Miškas f., 1 spec. on Fomes fomentarius on Alnus spp.

Rabdocerus gabrieli (Gerhardt, 1901)

Suginčiai, 29.09.2014, 1 spec., sweeping by net in a wet meadow.

\section{CHRYSOMELIDAE}

Bruchidius marginalis (Fabricius, 1771)

Branišavos Miškas f., 30.05.2016, 1 spec., 08.09.2016, 20 spec., 12.09.2016, 4 spec., 31.07.2018, 2 spec., sweeping by net in mesophytic meadows; Suginčiai, 1978, 1 spec., 18.08.2014, 8 spec., 02.09.2014, 6 spec., 06.09.2014, 11 spec., 11.10.2014, 1 spec., most on Euphorbia cyparissias, also in mesophytic meadows.

Donacia antiqua Kunze, 1818

Lake Želva env. (2), 2006, 1 spec.

Hypocassida subferruginea (Schrank, 1776)

Suginčiai, 04-20.05.2007, $1 \mathrm{spec.}$

Chrysolina aurichalcea (Gebler, 1825)

Vilemai, 20.07.2007, 1 spec. (det. V. T.)

Chrysolina graminis (Linnaeus, 1758)

Kapiniškès, 08.07.1995, $1 \mathrm{spec}$.

Chrysolina herbacea (Duftschmid, 1825)

Suginčiai, 06.07.2014, $2 \mathrm{spec}$, in a vegetable garden in Mentha arvensis overgrowth. Later, from end-May to mid-October, 2015-2018, 20-60 adults and larvae were constantly recorded. 
Colaphus sophiae (Schaller, 1783)

Suginčiai, 08.05.2016, 1 spec., in a garden (det. R. F., V. T.).

Sermylassa halensis (Linnaeus, 1767)

Branišavos Miškas f., 29.09.2015, 3 spec., 29.08.2018, 1 spec.; Kašeikiai, 13.08.2014, near 10 spec., 08.09.2014, 6 spec,, 30.09.2014, near 20 spec.; Suginčiai, 31.07-05.09.2014, about 50 spec., 06.08-26.09.2015, about 20 spec.; Lake Želva env. (1), 05.09.2014, 1 spec. On Euphorbia cyparissias, thin on Convolvulus arvensis or other plants in mesophytic meadows.

Galeruca interrupta (Illiger, 1802)

Lake Obelija env., 06.06.1996, 2 spec. (det. V. T.).

Prasocuris junci (Brahm, 1790)

Kapiniškès, 21.07.2018, 2 spec., on Veronica beccabunga. One report (Tamutis, 2003).

Hermaeophaga mercurialis (Fabricius, 1792)

Kulionys env., 16.06.2015, 1 spec.; Suginčiai, June 2007, 1 spec.; Verkiai Park, 18.05.2015, about 600,000 spec., in Mercurialis perennis habitats in an area of about 3.5 ha.

Note. Species abundance in Verkiai Park, Vilnius environs, was estimated using the technique of sampling plots, with adults being counted in $1 \mathrm{~m}^{2}$ (10 plots) in different places. The species is rare in Lithuania, with the first record made in 1991 (Pileckis, 1997). The case of exponential growth of the population of this rare species was registered, which is particularly rare in natural conditions. In 1991, the exponential growth of the species probably reached the maximums of population density and abundance. A repeated survey was carried out in the same locality in 2017; however, not a single specimen of the species was detected.

Phyllotreta tetrastigma (Comoli, 1837)

Suginčiai, 18.05.2015, 1 spec.; Lake Želva env. (1), 19.05.2015, about 50 spec., on Cruciferae on the lakeshore.

\section{Altica brevicollis Foudras, 1860}

Branišavos Miškas f., 25.09.2016, 5 spec.; Lake Želva env. (2), 08.05.2016, 6 spec., 06.06.2017, 3 spec., on Corylus avellana.

Labidistomis tridentata (Linnaeus, 1758)

Branišavos Miškas f., 14.06.2015, 1 spec.; Suginčiai, 15.06.2015, 12-21.06.2016, 17.06.2017, 20 spec., on Betula spp., Quercus robur, Salix spp.
Cryptocephalus coryli (Linnaeus, 1758)

Ažuolijos Miškas f., 23.05.2006, 1 spec., on Sorbus aucuparia blossoms.

Cryptocephalus pini (Linnaeus, 1758)

Lake Želva env. (2), 22.08.2017, 1 spec., by a pheromone trap for Ips typographus control.

Cryptocephalus nitidus (Linnaeus, 1758)

Lake Želva env. (1), 18.04.2014, 1 spec., 21.06.2016, 1 spec.

\section{ANTHRIBIDAE}

Gonotropis dorsalis (Thunberg, 1796)

Lake Želva env. (2), 11.08.2017, 1 spec., by a pheromone trap for Ips typographus control.

Rhaphitropis marchicus (Herbst, 1797)

Branišavos Miškas f., 10.06.2015, 1 spec., reared from Alnus spp. One report (Tamutis, 2003).

\section{Platyrhinus resinosus (Scopoli, 1763)}

Suginčiai, 21.05.2017, 1 spec., 16.06.2017, 4 spec., 13.06.2018, 2 spec.; Kulionys env., 07.06.2015, 1 spec., on Alnus spp., Betula spp.

\section{DISCUSSION}

As many as eleven genus Medon (Hypomedon, Sunius, Luzea) species are widely distributed in Middle and Northern Europe; however, they are encountered sporadically with only solitary specimens recorded, their "ecology is nearly unknown, at least some (hopefully, all) live in underground nests of mouse-like rodents or moles" (Lompe A., after Lohse G. A.). In Europe, Medon apicalis was registered in Austria, the Czech Republic, Greece, Croatia, Macedonia, all Scandinavian countries, and Germany. Quite recently, one specimen of the species has been recorded in England and several specimens in western Poland (Assing et al., 2011; Burakowski et al., 1979; Fauna Europaea Database; Lompe, 2018). The locality where Medon apicali was recorded in Lithuania is the easternmost edge of the species distribution range in Europe.

Xylodromus depressus and Heterothops dissimilis are widely distributed in Europe. They have been found in nearly all Middle and Northern European countries (Fauna Europaea Database). Both species are predators that hunt under the bark of dead deciduous trees (Assing et al., 2011). 
Abdera flexuosa, a xylomicetopagous beetle, lives in soft wood partly decomposed by rot fungi (Kaszab in: Freude et al., 1969; Duff, 2012). The author found it only in birch trees (Betula spp.) growing in areas surrounding swamps, or in damp forests.

The representatives of Histeridae, Eucnemidae, Elateridae, Lymexylidae, Trogossitidae, Silvanidae (except Ahasverus advena), Endomychidae, Mycetophagidae, Tetratomidae, Melandryidae, Zopheridae, Tenebrionidae (except Blaps mortisaga), Oedemeridae, Boridae, Salpingidae, and Anthribidae families described in the list of species are xylobionts (saproxyls in broad sense), with many species included into the EU lists of protected species (Nieto et al., 2010). However, more comprehensive studies, in particular the maintaining larvae and rearing adult beetles in laboratory, showed that some published ("rare") species such as: Abdera affinis, Aspidiphorus orbicularis, Cyllodes ater, Dendrophilus punctatus, Dorcatoma dresdensis, Epuraea boreella, Grynocharis oblonga, Hallomenus binotatus, Hylleocoetus dermestoides, Leiopus linnei, Glischrochilus quadriguttatus, Megatoma undata, Mordellistena abdominalis, Mycetochara flavipes, Mycetophagus piceus, M. quadriguttatus, M. multipunctatus, Nitidula bipunctata (by tree sap), Oplocephala haemorrhoidalis (northeastern Lithuania), Orchesia micans, Peltis grossa, Pocadius adustus, Salpingus ruficollis, Soronia grisea, Synchita humeralis, and Tetropium fuscum are widespread and can be met nearly everywhere in their suitable habitats or microhabitats. Saproxylic beetles constitute an ecological group of beetles (Coleoptera) that are insufficiently investigated in Lithuania, and so far no publication has emerged on laboratory studies of this abundant group of the species.

Long-term investigations disclosed interesting facts about the ecology of some species, namely: the records of Calosoma auropunctatum in the depth of the forest for several years (in European literature this species is described as living in open areas); exponential growth of Hermaeophagus mercurialis population; records of Boros schneideri deep in raised bogs, in open areas with solitary pine trees.
According to different European literature, Agostenus costulatus, Eubria palustris, Silvanoporus fagi, Leiestes seminiger, Mycetophagus ater, Orchesia undulata, Anogcodes ferrugineus, Anogcodes rufiventris, and Lissodema cursor are regarded as rare and local (fragmentary records, isolated populations) in Europe, while Anthobium unicolor, Oxyporus mannerheimi, Isoriphis melasoides, Drapetes mordelloides, Nemozoma elongatum, Physoronia wajdelota, Plegaderus saucius, Wanachia triguttata, and Phyllotreta tetrastigma are reported as rare and insufficiently known (with scanty data on their distribution, abundance, and other aspects of species life).

\section{ACKNOWLEDGEMENTS}

The author appreciates the help of the Laboratory of Entomology of the Nature Research Centre in preparing this paper and expresses his thanks to Pavelas Starkevičius and Radvilè Markevičiūte for photos of beetles, and to Dr. Romas Ferenca, Vidmantas Monsevičius, and Assoc. Prof. Vytautas Tamutis for assistance in identifying some beetle species. The author is grateful to Prof. Vincas Būda for his suggestions in preparing this paper.

Received 18 March 2019 Accepted 25 March 2019

\section{References}

1. Assing V, Schulke M. Die Kafer Mitteleuropas. Band 4: Staphylinidae. Heidelberg. 2011. German.

2. Burakowski B. Klucze do oznaczania owadow Polski. XIX (88-90). Pythidae, Lagriidae, Alleculidae. Warszawa. 1976. Polish.

3. Burakowski B, Mroczkowski M, Stefanska J. Katalog fauny Polski, T. 7: Chraszcze - Coleoptera. Kuskowate - Staphylinidae. Warszawa. 1979. Polish.

4. Duff AG, editor. (after Hackston M.) 2014. Checklist of beetles of British Isles, Melan- 
dryidae.Available from http://www.coleoptera.org.uk/sites/www.coleoptera.org.uk/ files/imce/coleoptera/checklist2012.pdf.

5. Ferenca R. 9 new for Lithuania species of beetles, found in 1981-1985. In Jonaitis V. (ed.). New and Rare for Lithuania Insect Species. Records and Descriptions of 1987. Vilnius: 1988; 22-26.

6. Ferenca R, Ivinskis P, Meržijevskis A. New and rare Coleoptera species in Lithuania. Ekologija. 2002; 3: 25-31.

7. Ferenca R, Ivinskis $\mathrm{P}$, Meržijevskis A, Rimšaitè J, Tamutis V. New data on Lithuanian beetle (Coleoptera) fauna. New and Rare for Lithuania Insect Species. 2008; 20: 29-38.

8. Ferenca R, Tamutis V. $17 \mathrm{New}$ for Lithuanian fauna species of beetles (Coleoptera). New and Rare for Lithuania Insect Species. 2009. 21: 32-9.

9. Feude H, Harde KW, Lohse GA. Die Kafer Mitteleuropas. Bds. 2-16. Krefeld, Germany: Goecke and Evers. 1965-1989.

10. Lompe A. (after Lohse G. A.). 2018. Kafer Europas, Medon, Coleoptera-StaphylinidaePaederinae. Available from http.//www. coleonet.de/coleo/medon/coleoptera.htm. [cited 2018 Oct).

11. Monsevičius V. New and little known for the Lithuanian fauna species of beetles (Coleoptera), found in 2002, 2011-2012. New and Rare for Lithuania Insect Species. 2013; 25: 24-31.

12. Nieto A, Alexander K. European Red List of Saproxylic Beetles. Publication Office of the European Union. Luxenbourg. 2010; 46.

13. Pacevičius V. New for Lithuanian fauna species of beetles (Coleoptera) found in Molètai district. Bulletin of the Lithuanian Entomological Society. 2017; 1(29): 27-9.

14. Pileckis S, Monsevičius V. Lietuvos fauna. Vabalai 2. Vilnius, Mokslas. 1997. Lithuanian.

15. Tamutis V. Eighty-two new for Lithuania beetle (Coleoptera) species. New and Rare for Lithuania Insect Species. 2003; 15: 54-62.
16. Tamutis V, Tamute B, Ferenca R. A Catalogue of Lithuanian beetles (Insecta, Coleoptera). Zookeys. 2011; 121: 1-464. Sofia.

17. WarchałowskiA.Kluczedooznaczania owadow Polski. XIX (94 a). Stankowate - Chrysomelidae. Warszawa, 1971. Polish.

\section{Viktoras Pacevičius}

\section{MAŽAI ŽINOMOS ARBA LIETUVOJE RETOS NAUJOS VABALŲ (COLEOPTERA) RŪŠYS IR KAI KURIE JU EKOLOGIJOS BRUOŽAI}

\section{Santrauka}

Pateikiami duomenys apie 120 retu arba mažai žinomų vabalų (Coleoptera) rūšių, iš kurių viena, Medon apicalis, yra nauja Lietuvoje. Agostenus costulatus, Anogcodes ferrugineus, Anogcodes rufiventris, Eubria palustris, Leiestes seminiger, Lissodema ursor, Mycetophagus ater, Orchesia undulata, Sivanoporus fagi Europoje yra retos ir lokalios, Anthobium unicolor, Drapetes mordelloides, Isoriphis melasoides, Oxyporus mannerheimi, Phyllotreta tetrastigma, Physoronia wajdelota, Plegaderus saucius, Nemozoma elongatum, Wanachia triguttata - retos bei mažai žinomos. Nustatyta, jog saproksilinès rūšys Abdera flexuosa, Hallomenus axillaris, Hyleocoetus flabellicornis, Lissodema cursor, Orchesia undulata, Triphyllus bicolor, Wanachia triguttata yra ksilomicetofagai, gyvenantys trūnijančiuose, grybais apaugusiuose medžiuose arba medžių grybų vaisiakūniuose. Išaiškèjo nauji trijų rūšių ekologijos faktai: Calosoma auropunctatum populiacija rasta 1994, 1996 ir 1998 metais brandaus mišraus miško gilumoje; registruota gausi Hermaeophagus mercurialis populiacija (apie 600000 individų maždaug 3,5 ha plote); Boros schneideri gyvena žuvusiose be žievès pušaitèse aukštapelkių gilumoje. Pirma Lietuvoje Medon apicalis radimviete yra labiausiai į rytus nutolęs rūšies radimo taškas Europoje.

Raktažodžiai: vabzdžiai, saproksilinès rūšys, ksilomicetofagai, Medon apicalis, Boros schneideri 